\title{
Précision de la problématique
}

Swiss Medical Board

Correspondance:

Susanna Marti Calmell

Secrétariat de l'organe responsable du Swiss

Medical Board

Stampfenbachstrasse 30

$\mathrm{CH}-8090$ Zurich

Tél. 0432595211

info[at]medical-board.ch

www.swissmedicalboard.ch

Les prises de position concernant le rapport «Traitement oral ou parentéral de la carence en fer", parvenues dans le cadre de la post-publication-review, portent en particulier sur la clarté de la problématique présentée. Le Swiss Medical Board a précisé la formulation de la problématique du rapport publié en octobre 2014.

Après la publication du rapport «Traitement oral ou parentéral de la carence en fer", l'organe responsable du Swiss Medical Board avait invité toutes les parties intéressées à s'exprimer dans le cadre d'une post-publication-review. La majorité des réponses ont souligné que le diagnostic, respectivement le groupe de patients faisant l'objet du rapport, ne ressortait pas assez clairement du rapport et que la définition de l'expression "carence en fer sévère symptomatique» manquait de précision.

Le rapport analyse le traitement d'une carence en fer sévère symptomatique, dont les principaux symptômes sont une fatigue et un abattement extrêmes. Il s'agit, en l'occurrence, de la question de savoir si, par rapport à un traitement oral, un traitement parentéral (intraveineux) apporte un avantage au patient en termes d'efficacité et de qualité de vie.

Le présent rapport ne concerne pas le traitement de la carence en fer sans symptômes. Il renonce également à proposer une définition précise de la "carence sévère en fer", celle-ci ne pouvant pas se référer à des valeurs de laboratoire universelles. Toutefois, le rapport part du principe qu'une carence en fer sévère comprend les stades II «carence en fer avec troubles initiaux de l'hématopoïse» ( $\mathrm{Hb}$ normal, $\mathrm{MCV}$ et $\mathrm{MCH}$ faibles) et III «anémie ferriprive» (Hb faible). Par ailleurs, le rapport se limite à la forme de la thérapie - administration orale, plus simple et moins onéreuse, ou administration par voie intraveineuse plus chère - lorsque l'indi- cation pour la substitution est incontestée. L'indication pour le traitement de la carence en fer n'est pas analysée.

\section{Les conclusions restent identiques}

Les réactions émanant du corps médical ont convaincu le groupe d'experts de réviser le rapport quant aux points abordés. L'accent sur la "carence en fer sévère symptomatique» ressort désormais du titre du rapport. Les conclusions et les recommandations du rapport restent néanmoins identiques:

- Chez les patients présentant une carence en fer sévère symptomatique ou une anémie ferriprive, un traitement substitutif parentéral est souhaitable après un examen minutieux. Il convient, pendant l'administration du traitement, d'assurer une surveillance adéquate et d'être prêt à intervenir par rapport à d'éventuels effets secondaires.

- Il convient, dans le même temps, de mettre en place un traitement adéquat de la / des cause(s) de la carence en fer.

- Il importe, en outre, de toujours vérifier si les patients peuvent bénéficier d'un traitement parentéral de substitution en fer dans le cadre d'une intervention chirurgicale.

Le rapport concernant la post-publication-review et le rapport révisé sont publiés sur le site internet du SMB. 